\title{
Relacionamento interpessoal, produtividade e habilidades sociais: um estudo correlacional
}

\author{
Jucelaine Bier Di Domenico Grazziotin - Universidade de Passo Fundo, Passo Fundo/RS, Brasil \\ Silvana Alba Scortegagna - Universidade de Passo Fundo, Passo Fundo / RS, Brasil
}

\begin{abstract}
Resumo
O presente estudo buscou evidências de validade do Zulliger, focalizando as variáveis relacionamento e produtividade, em correlação com o Inventário de Habilidades Sociais - IHS. A amostra foi composta por 40 sujeitos, entre 18 e 43 anos, funcionários de uma empresa. Entre os indicadores, as variáveis GHR, GPHR, Pure H e SumH correlacionaram-se positiva e significativamente com os fatores GIHS, F3, F5 e F1. Contrariamente, as variáveis $A G$ e PHR apresentaram correlações significativamente negativas com o F5. O número de respostas $R$ não obteve correlação significativa com os fatores do IHS-Del-Prette, do ponto de vista estatístico, mas qualitativamente denotou conexão. Os resultados contribuíram para assegurar a validade do ZSC e confirmaram outras expectativas do estudo ao demonstrar um bom relacionamento interpessoal e uma boa produtividade entre os participantes.

Palavras chave: Avaliação psicológica; Habilidades sociais; Teste de Zulliger; Validade.
\end{abstract}

Interpersonal relationships, productivity and social skills: a correlational study

\begin{abstract}
This study intended to verify the validity evidence of the Zulliger, focusing on the relationship and productivity variables in correlation with the Social Skills Inventory HIS - Del-Prette. The sample was composed of 40 subjects between 18 and 43 years of age, employees of a company. Among the indicators, the variables GHR, GPHR, Pure H and Sum H had significant positive correlations with factors GIHS, F3, F5 and F1. On the other hand, the variables AG and PHR demonstrated significant negative correlation with $\mathrm{F} 5$. The number of responses $\mathrm{R}$ had no significant relation with the factors of IHS-Del- Prette, but it showed a qualitative connection. The results contributed to ensure the validity of ZSC and confirmed other expectations of the study by demonstrating a good productivity and relationship with the participants.

Keywords: Psychological assessment; Social skills; Zulliger test; Validity
\end{abstract}

Relación interpersonal, productividad y habilidades sociales: um estudio de correlación

\begin{abstract}
Resumen
Este estudio buscó evidencias de validez del test de Zulliger, con énfasis en las variables de relación y productividad, en correlación con el Inventario de Habilidades Sociales - IHS. Para componer la muestra participaron 40 sujetos, entre 18 y 43 años, empleados de una empresa. Entre los indicadores, las variables GHR, GPHR, Pure H y SumH se correlacionaron positivamente y de manera muy significativa con los factores GIHS, F3, F5 y F1. Sin embargo, las variables AG y PHR presentaron correlaciones negativas con el F5. El número de respuestas $\mathrm{R}$ no obtuvo correlación significativa con los factores del IHS-Del-Prette, desde el punto de vista estadístico, pero de manera cualitativa, se percibió una conexión. Los resultados contribuyeron para garantizar la validez del ZSC, y confirmaron otras expectativas de la investigación mientras que demostraron una buena relación interpersonal y una buena productividad entre los participantes.

Palabras-clave: Evaluación psicológica; Habilidades sociales; Test de Zulliger; Validez.
\end{abstract}

Nas últimas décadas as diretrizes do Conselho Federal de Psicologia (CFP) voltaram-se fortemente para o estabelecimento de ações técnicas e políticas que amparassem a prática científica da avaliação psicológica e o uso de testes. Entre essas, a resolução no 002/2003 versa sobre o uso, a elaboração e a comercialização de testes; a resolução $\mathrm{n}^{\circ}$ 07/2003 institui o manual de elaboração de documentos; e a resolução no 010/2005 regulamenta o código de ética profissional. Além disso, o uso dos testes no Brasil passou a ser regulado por um grupo de especialistas e conselheiros que compõem o Sistema de Avaliação dos Testes Psicológicos (SATEPSI).

Essa comissão avalia se os testes apresentam, ou não, as condições técnicas mínimas para o uso profissional, em relação a quatro critérios: fundamentação teórica, precisão, validade e sistema interpretativo (Nunes, 2011; Nunes \& Primi, 2010). Um dos objetivos desse rigoroso trabalho é garantir que o examinador disponha de instrumentos com padrão de qualidade para que possa obter informações seguras.
Sob essa perspectiva, há necessidade constante de estudos de validade dos instrumentos, nos mais diversos contextos, inclusive no campo empresarial. As práticas avaliativas nesse âmbito revelam que os examinandos, especialmente em situações de seleção, podem comportar-se melhor do que são e responder aos testes de autorrelato da maneira mais condizente com as expectativas do empregador (Dilchert, Ones, Visweswaran \& Deller, 2006; Meade, 2004). Daí, ser comum a escolha de testes projetivos para essa finalidade.

Nessas condições, o Zulliger no Sistema Compreensivo - ZSC mostra-se uma ferramenta oportuna. O teste permite, de modo indireto, uma avaliação mais completa do funcionamento psíquico do indivíduo, pois possibilita a interpretação dos indicadores, das variáveis que compõem o Sumário Estrutural, de forma psicométrica e projetiva (Villemor-Amaral \& Primi, 2009).

As variáveis que formam o agrupamento do relacionamento interpessoal desse sistema, particularmente, são relevantes. Elas representam as 
necessidades, as atitudes, os estilos de comunicação que o sujeito mantém com os demais. Como a relação interpessoal é um dos elementos essenciais e constitutivos da conduta humana, é, também, um dos campos onde mais facilmente se produzem os conflitos, convertendo-se em uma área de exame obrigatória para a compreensão do funcionamento mental (Exner \& Sendín, 1999).

Outro índice importante a ser observado em um protocolo é o número de respostas $R$. $O R$ faz parte de outro agrupamento de variáveis do ZSC, o de recursos e controle, e está sendo considerado, no presente estudo, por mensurar a capacidade de produtividade, motivação, produção de ideias, energia, colaboração (Villemor-Amaral \& Primi, 2009). Segundo Exner (2003), o R também pode ser associado a alguns estilos de personalidade, e quando reduzido pode indicar inibições, limitações intelectuais, componentes depressivos, traços paranoides, fóbicos, além de resistências, desinteresse ou preparo inadequado para realizar o teste.

Mediante o exposto, alguns estudos mostram a relevância do ZSC em diversas conjunturas. Zudnic (2003), na Argentina, elaborou listas de qualidade formal e respostas populares, e estabeleceu normas para interpretação das variáveis do sumário estrutural. A autora analisou 300 protocolos, de sujeitos entre 18 e 55 anos, avaliados em circunstâncias de seleção de pessoal, o que trouxe contribuições para a normatização e validação do ZSC neste contexto.

Fazendeiro e Novo (2012), em Portugal, exploraram as potencialidades do ZSC com uma amostra de 156 militares do exército, em aplicação coletiva. Os resultados mostraram-se positivos, mas evidenciaram a necessidade de um padrão de aplicação, de novos estudos normativos e de validação para diferentes contextos.

No Brasil, houve avanços importantes em estudos de validação, sobretudo a partir de 2005. Ferreira e Villemor-Amaral (2005) buscaram a correlação do ZSC com um questionário de avaliação de desempenho de uma empresa, com 86 sujeitos adultos, profissionais das ciências exatas. As correlações foram positivas e indicaram uma predição do bom desempenho dos profissionais: $X+\%$ se correlacionou com solução de problemas; $D d$ se correlacionou negativamente com a capacidade para solucionar problemas; e o aumento de $Y$ demonstrou a presença de pessoas com elevado estresse situacional e características depressivas.

Motivadas por objetivo semelhante, Di Domenico-Grazziotin e Scortegagna (2012) investigaram a correlação entre a variável do relacionamento do ZSC e o Inventário de Habilidades Sociais IHS-Del-Prette (Del Prette \& Del Prette, 2005). A amostra constitui-se de 19 sujeitos adultos, funcionários de uma empresa comercial. Foram encontradas correlações positivas estatisticamente significativas nas variáveis GHR, GPHR, pure $H$, Sum $H$ do ZSC com os fatores GIHS, F3 e F1 do IHS, e correlações negativas estatisticamente significativas nas variáveis $A G$, PHR, Sum $T$ do ZSC com os fatores GIHS e F5. Os achados exibiram correlações expressivas em diversos indicadores do ZSC, o que contribuiu para asseverar a sua validade.

Três estudos de convergência entre o ZSC e o Pfister foram realizados por Franco e Villemor-Amaral (2009), com amostras de pacientes psiquiátricos e de não pacientes. $O$ primeiro, buscou evidenciar a validade das constelações do ZSC em uma amostra de 153 indivíduos; o segundo, correlacionou os indicadores afetivos e cognitivos em 223 protocolos e o terceiro, averiguou a validade de critério ao comparar as respostas emitidas em 40 protocolos. O estudo ampliou a compreensão de aspectos da personalidade, e dos alcances e limites das técnicas projetivas. Nesse mesmo ano, também, Franco, Cardoso, VillemorAmaral e Primi (2009) desenvolveram estudos de normatização do ZSC para a população brasileira; participaram 475 pessoas.

Posteriormente, Santana, Cardoso e VillemorAmaral (2011) e Villemor-Amaral e Cardoso (2011) seguiram com os estudos de validade do Zulliger. No primeiro, as pesquisadoras procuraram instituir a Nota $Z$, existente no Sistema Compreensivo de Exner (2003), no teste de Zulliger na Argentina (Zudnic, 2003), mas não sendo, ainda, utilizada no Brasil. Para isso, foram analisados 50 protocolos de uma base de dados pertencentes aos autores do manual do Teste de Zulliger ZSC. No segundo, com o intuito de verificar a validade do tipo de vivência (EB), as autoras utilizaram um banco de dados com 51 protocolos de adultos com características sociodemográficas variadas. Foram realizadas correlações entre o número de respostas $\mathrm{Me}$ o escore WSumC de ambos os testes. Os dados sugeriram que o Zulliger e o Rorschach produzem respostas $\mathrm{M}$ de modo mais semelhante do que as respostas de cor.

Recentemente, Franco e Villemor-Amaral (2012), buscaram a validade incremental do teste de Zulliger e do Pfister, a partir da compreensão de 20 dependentes químicos. As autoras realizaram uma avaliação da personalidade de 10 brasileiros adictos de álcool e de 10 franceses dependentes da heroína. Os resultados ratificaram as informações geradas pelos instrumentos.

Apesar de algumas pesquisas demostrarem a relevância do ZSC em diversas situações e demandas, os estudos ainda são escassos. Recentemente, Burlamaque, Zardo, Secco e Scortegagna (2012) efetuaram um levantamento nas bases de dados BVSPSI (SciELO, PePSI, LILACS e RedALCYC) dos artigos publicados nos periódicos brasileiros, no período de 2002 a 2012, e encontraram apenas 13 estudos. Outro instrumento que objetiva avaliar um construto semelhante ao do relacionamento interpessoal do ZSC é o Inventário de Habilidade Social IHS-Del-Prette (Del Prette \& Del Prete, 2005). 
Esses autores associam essa habilidade com a produtividade e referem que indivíduos menos conflitados apresentam melhor competência social. Tais habilidades refletem um desempenho socialmente competente e podem ser aprendidas e alteradas ao longo da vida (Del Prette \& Del Prette, 2010).

Entre os estudos relacionados, Bandeira, Costa, Del Prette, Del Prette e Gerk-Carneiro (2000) buscaram a validade do IHS-Del-Prette com uma amostra de 104 estudantes de psicologia. Os resultados foram qualificados como válidos e confiáveis tanto para avaliação diagnóstica quanto para efeitos de intervenção.

Dois estudos com o IHS-Del-Prette merecem destaque. $\mathrm{O}$ primeiro analisou o repertório de habilidades sociais em 10 profissionais de uma empresa. Os resultados confirmaram a importância dessas habilidades para o bem-estar e produtividade dos funcionários (Silva, 2004). O segundo investigou a relação entre habilidades sociais e bem-estar psicológico de 232 jovens, em situação de desemprego. A análise dos dados revelou correlação significativa entre os dois construtos (Guilland \& Monteiro, 2010).

Outra pesquisa conduzida por Angélico, Crippa e Loureiro (2012) objetivou verificar as associações entre as manifestações clínicas do Transtorno de Ansiedade Social (TAS) e aferir a validade discriminativa do IHSDel-Prette com 1.006 universitários. Os resultados indicaram que quanto mais elaborado for o repertório de habilidades sociais, menor será a probabilidade de diagnóstico para o TAS.

Considerando a escassez de estudos de validade do ZSC e as consequências negativas que tal carência acarreta para manter o caráter científico da avaliação psicológica e para a prática profissional, constitui-se objetivo deste estudo buscar evidências de validade do ZSC, focalizando as variáveis relacionamento e a produtividade, em correlação com os fatores do IHSDel-Prette (Del Prete \& Del Prete, 2005). Entre as hipóteses, supõe-se que as variáveis que informam sobre o bom relacionamento e percepção interpessoal adequada se correlacionem positiva e significativamente com fatores do IHS-Del-Prette, e as variáveis que indicam dificuldades de relacionamento, apresentem correlações negativas com o IHS-DelPrette. Além disso, espera-se encontrar alguma ligação positiva entre o número de respostas e os fatores do IHS-Del-Prette.

\section{Método}

\section{Participantes}

Foram participantes 40 sujeitos, entre 18 e 43 anos de idade, média 26,5 anos (DP=7,99), 23 (57\%) do sexo feminino e $17(43 \%)$ do sexo masculino, 34 (85\%) com ensino médio completo, de uma das lojas de uma rede de supermercados, no Rio Grande do Sul. Os participantes foram selecionados de forma intencional, sendo incluídos os que exerciam atividades de atendimento direto ao cliente: 18 (45\%) operadores e frente de caixa, $14(35 \%)$ atendentes de perecíveis e 8 $(20 \%)$ atendentes de loja.

\section{Instrumentos}

Zulliger no Sistema Compreensivo ZSC - forma individual (Villemor-Amaral \& Primi, 2009) composto por um jogo de três cartões, com manchas de tinta não estruturadas, que possibilitam, por meio do agrupamento de variáveis, compreender o funcionamento psíquico do indivíduo. Quanto à sua confiabilidade, foram realizados dois estudos de precisão, um empregando o teste-reteste e o outro analisando a precisão do avaliador. No teste-reteste (Villemor-Amaral; Machado \& Noronha, 2009), a maior parte dos indicadores alcançou valores acima de $0,70(\mathrm{p}=0,001)$; os resultados de concordância entre os juízes revelaram correlações entre $0,60-0,80$, indicando uma associação alta (Muniz, Machado, VillemorAmaral \& Primi, 2009). A administração do teste consiste na fase da associação, em que o sujeito deve responder à questão "com que isso se parece"; e na fase do inquérito, onde se verifica "onde foi que a pessoa viu" e "o que na mancha fez com que parecesse aquilo" que foi dito.

Neste estudo, além do $R$ (número de respostas), estão sendo consideradas as variáveis que compõem o relacionamento, a saber: $\operatorname{Sum} T$ (sombreado textura); Fd (comida ou ação de comer); $H$ puro (humano inteiro), Sum $H$ (soma de $H$ puro), $H d$ (detalhe humano), (H) (para-humano inteiro), (Hd) (detalhe para-humano), GHR (boa representação humana) e PHR (representação humana pobre), GPHR (proporção de boas respostas humanas e pobres), a (movimento ativo), $p$ (movimento passivo), isolate (isolamento), PER (resposta personalizada), $A G$ (movimento agressivo), COP (movimento cooperativo).

Inventário de Habilidades Sociais - IHS-Del-Prette_(Del Prette \& Del Prette, 2005) - instrumento de autorrelato, com 38 itens, que avaliam as habilidades sociais. É um teste com padrão de confiabilidade e de consistência interna (alfa de Cronbach $=0,75$ ); estabilidade teste-reteste $(\mathrm{r}=0,90 ; p=0,001)$ e validade concomitante com o Inventário de Rathus $(\mathrm{r}=0,79 ; p=0,01)$ (Bandeira, Costa, Del Prette, Del Prette \& Gerk-Carneiro, 2000). Nas instruções, o respondente deve estimar a frequência com que reage a uma situação descrita em cada item, em uma escala tipo likert, que varia de 0 (nunca ou raramente) a 4 (sempre ou quase sempre), que avalia cinco fatores relacionados à habilidade social. $\mathrm{O}$ escore fatorial 1 (F1) mostra $\mathrm{O}$ repertório em habilidades de enfrentamento com risco. O escore fatorial 2 (F2) exibe habilidades de auto afirmação na expressão de afeto positivo. O Escore Fatorial 3 (F3) reúne habilidades de conversação e desenvoltura social. O escore fatorial 4 (F4) é um indicador de habilidades de auto exposição a 
desconhecidos ou a situações novas. O escore fatorial 5 (F5) avalia as habilidades de auto controle da agressividade em situações aversivas.

\section{Procedimentos}

Após a aprovação pelo Comitê de Ética em Pesquisa (n. 076/2009), iniciou-se a coleta de dados. O contato com o responsável de uma das lojas possibilitou a indicação dos participantes. Todos os funcionários, responsáveis por setores de atendimento direto ao público, foram convidados a participar do estudo, e aceitaram o convite. Aos participantes foram expostos os objetivos e os procedimentos do trabalho e, mediante a assinatura do Termo de Consentimento Livre e Esclarecido, procedeu-se às instruções do ZSC seguido pelo IHS-Del-Prette, de forma individual, em uma sessão de duas horas. A coleta de dados deu-se nas dependências da empresa, pela autora da pesquisa e por uma aluna do último ano do curso de Psicologia.

As respostas do ZSC foram codificadas e digitadas em um banco de dados do programa SPSS, versão 11.5. para a realização de análises estatísticas. $\mathrm{Na}$ correlação entre os resultados do ZSC e os do IHSDel-Prette, utilizou-se a prova de Pearson. Foi adotado como nível de significância um valor de $p<0,05$. Os escores foram confrontados com as referências normativas do grupo de não pacientes de VillemorAmaral e Primi (2009) para o ZSC, e de Del Prette e Del Prette (2005) para o IHS-Del-Prette.

\section{Resultados}

$\mathrm{Na}$ descrição dos resultados serão apresentadas as estatísticas descritivas das variáveis de relacionamento (Sum T, Fd, H, Sum H, GHR e PHR, a e p, isolate, PER, $A G, C O P)$ e do número de $\mathrm{R}$ do $\mathrm{ZSC}$ e, na sequência, as do IHS-Del-Prette. Por fim, apresentam-se as análises de correlação entre ambos. Dessa forma, na Tabela 1, podem ser visualizadas as estatísticas descritivas do ZSC.

Tabela 1. Estatísticas descritivas das variáveis de relacionamento e do número de respostas do ZSC

\begin{tabular}{|c|c|c|c|c|c|c|c|c|}
\hline Zulliger & Mín. & Máx. & Média & Mediana & $\mathrm{DP}$ & 25 & 50 & 75 \\
\hline $\mathrm{COP}$ & 0 & 2 & 0,60 & 1,00 & 0,63 & 0 & 1 & 1,00 \\
\hline $\mathrm{AG}$ & 0 & 2 & 0,48 & 0,00 & 0,68 & 0 & 0 & 1,00 \\
\hline GHR & 0 & 3 & 1,35 & 1,00 & 0,89 & 1 & 1 & 2,00 \\
\hline PHR & 0 & 3 & 0,88 & 1,00 & 0,91 & 0 & 1 & 1,75 \\
\hline GPHR & -2 & 3 & 0,48 & 0,00 & 1,26 & 0 & 1 & 1,00 \\
\hline$a-p$ & -1 & 5 & 0,68 & 1,00 & 1,87 & -1 & 1 & 2,00 \\
\hline $\mathrm{Fd}$ & 0 & 2 & 0,15 & 0,00 & 0,43 & 0 & 0 & 0 \\
\hline SumT & 0 & 2 & 0,48 & 0,00 & 0,68 & 0 & 0 & 1,00 \\
\hline SumH & 0 & 6 & 1,90 & 1,50 & 1,39 & 1 & 1,5 & 3,00 \\
\hline $\mathrm{H}$ & 0 & 3 & 1,00 & 1,00 & 0,82 & 0 & 1 & 1,00 \\
\hline PER & 0 & 2 & 0,58 & 0,00 & 0,78 & 0 & 0 & 1,00 \\
\hline Isolamento & 0 & 0,60 & 0,21 & 0,20 & 0,16 & 0,08 & 0,20 & 0,30 \\
\hline $\mathrm{R}$ & 5 & 14 & 8,40 & 8 & 2,15 & 7 & 8 & 9,75 \\
\hline
\end{tabular}

$\mathrm{Na}$ Tabela 1, pode-se observar que os participantes obtiveram uma média elevada em SumT $0,48(\mathrm{DP}=0,68)$ e uma média um pouco acima em COP 0,60 (DP=0,63), considerando os parâmetros normativos para SumT $0(\mathrm{DP}=0,2)$ e para COP 0,3
$(\mathrm{DP}=0,5)$. As demais variáveis de relacionamento do ZSC e o $R$ apresentaram-se entre as expectativas normativas. A Tabela 2 apresenta as estatísticas descritivas do IHS-Del-Prette.

Tabela 2. Estatísticas descritivas dos fatores do IHS

\begin{tabular}{lcccccccc}
\hline \multicolumn{1}{c}{ IHS } & Mínimo & Máximo & Média & Mediana & DP & 25 & 50 & 75 \\
\hline GIHS & 8 & 98 & 54,03 & 59 & 27,89 & 28,5 & 59 & 78 \\
F1IHS & 8 & 98 & 43,15 & 40,5 & 26,32 & 23 & 40,5 & 63 \\
F2IHS & 1 & 98 & 66,81 & 68 & 27,07 & 48 & 68 & 95,25 \\
F3IHS & 1 & 100 & 57,06 & 57,5 & 32,34 & 22,5 & 57,5 & 87,5 \\
F4IHS & 2 & 96 & 48,48 & 52,5 & 26,97 & 28,75 & 52,5 & 67,5 \\
\hline
\end{tabular}




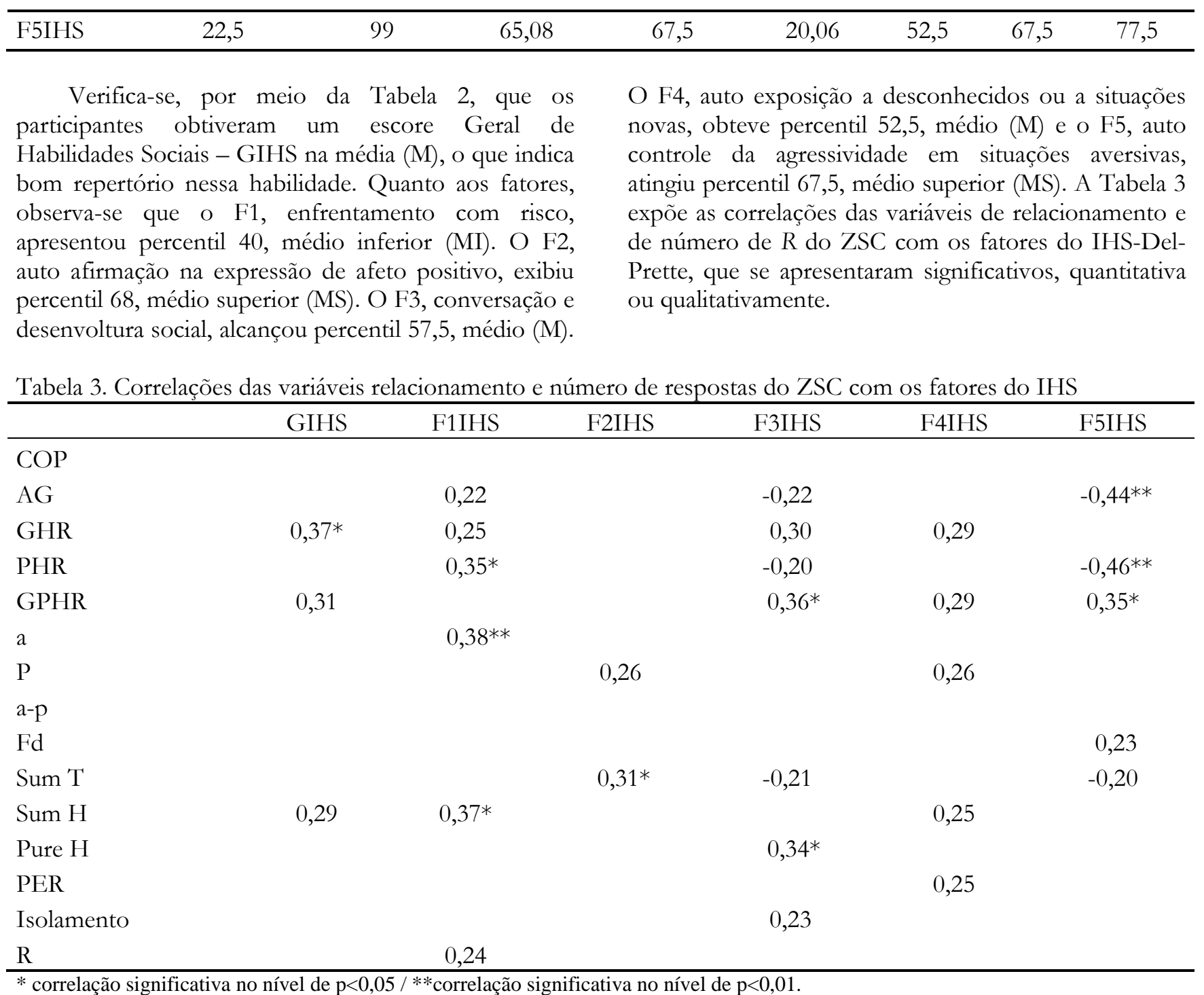

Pode-se visualizar, na Tabela 3 que as variáveis relacionamento do ZSC apresentaram correlações positivas ou negativas estatisticamente significativas de baixa ou moderada magnitude com o IHS-Del-Prete, tanto com o escore geral (GIHS), quanto com os fatores (F1, F2, F3, F4, F5). Observam-se correlações positivas significativas de GHR, GPHR, PURE $H$ e SumH com GIHS, F3, F5 e F1, respectivamente. Contrariamente, as variáveis, $A G$ e $P H R$ apresentaram correlações significativas e negativas com F5.

Quanto ao número de respostas do ZSC, não foram encontradas correlações significativas com os fatores do IHS-Del-Prette. Entretanto, na análise qualitativa, constatam-se questões interessantes, como se verá a seguir.

\section{Discussão}

Em relação ao ZSC, conforme a Tabela 1, a maioria das variáveis de relacionamento COP, $A G$ GHR, PHR, GPHR, a-p, Fd, Sum H, $H$ mantiveram-se na média normativa (Villemor-Amaral \& Primi 2009). Apenas as variáveis sombreado textura (SumT) e de movimento cooperativo (COP) apresentaram uma média um pouco mais elevada, de acordo com os parâmetros normativos.

Os índices que se mostraram nos valores normativos ilustram que os participantes tendem a apresentar percepções adequadas no que se refere ao modo de ver a si mesmos e aos outros (GHR, PHR, GPHR), ao interesse por pessoas (puro $H$ e Sum H), à propensão à iniciativa (a-p), à expectativa em relação aos outros $(F d)$, ao contato social (Isolate), à agressividade $(A G)$. Esses indicadores sugerem adequação no relacionamento interpessoal e no contato com os demais. As variáveis que apresentaram uma média um pouco elevada (Sum T e COP) indicam que o grupo possui avidez, necessidade de contato e uma propensão a perceber as relações pessoais de forma positiva (Villemor-Amaral \& Primi, 2009), características que condizem com as atividades laborais da amostra.

Esses achados asseguram os resultados do estudo preliminar de Di Domenico-Grazziotin e Scortegagna (2012), que observaram que a maioria das variáveis de relacionamento do ZSC mantiveram-se na média. As 
autoras igualmente encontraram índices um pouco mais elevados para Sum T.

De forma semelhante, o número de respostas $R$, que indica a quantidade total de respostas do protocolo, apresentou-se nos índices normativos. Desse modo, sujeitos ativos, produtivos, que confiam no entrevistador oferecem várias respostas. Tais pessoas podem, assim, ocupar-se de suas tarefas com apenas uma indicação geral acerca do que realizar (Zudnic, 2003). Nesse caso, os sujeitos em questão demonstraram um bom número de respostas, fato que também apoia e qualifica os outros índices obtidos, ou seja, se pode considerar que não houve uma tendência a inibições, limitações, desinteresses ou resistências diante da tarefa proposta, o que mostra colaboração e produtividade.

Assim como a maior parte das variáveis do relacionamento e o número de respostas no ZSC, também os fatores do IHS-Del-Prette mantiveram-se nos índices normativos. Por conseguinte, torna-se imprescindível discutir os escores dos fatores do IHSDel-Prette, que vem condizer com os resultados e as interpretações do ZSC.

É importante que os profissionais que trabalham com atividades de atendimento ao público, frequentemente expostos a condições estressantes, tenham um bom repertório de habilidades sociais, para que, assim, consigam realizar suas atribuições de uma forma produtiva. Para Del Prette e Del Prette (2005), as habilidades sociais têm ampla relação com a saúde mental, a satisfação pessoal, a realização profissional e a qualidade de vida, e refletem a capacidade de desempenho socialmente competente.

Observa-se que os participantes do grupo estudado apresentaram um repertório geral de habilidade social (GIHS), situado na média (M) (Tabela 2). Contudo, a análise dos fatores evidencia a presença de características próprias desse grupo. Esse aspecto pode ser verificado no F1, em que o grupo apresentou um repertório um pouco abaixo da média (MI), o que supõe uma atitude menos elaborada em ocasiões que demandam lidar com situações interpessoais que requerem a afirmação e defesa de direitos e autoestima, com risco potencial de reação indesejável (Del Prette \& Del Prette, 2005). Em compensação, no F2, a autoafirmação de afeto positivo, e, no F5, autocontrole da agressividade em situações aversivas, os resultados demonstraram a presença de um repertório elaborado (MS).

São muito interessantes esses achados, pois, além de asseverarem a validade do ZSC, vêm informar as características do grupo. São notórias as habilidades para lidar com demandas de afeto positivo e de afirmação da autoestima em situações que não envolvem riscos de reação indesejável, ou seja, o grupo parece agradecer elogios, expressar sentimentos positivos (F2) e possuir condições para reagir em situações aversivas com apropriado controle da raiva e da agressividade (F5). As elevações das variáveis Sum T e $C O P$, e o rebaixamento do AG em comparação com $C O P$ no ZSC confirmam essas características. Esses dados vêm, outra vez, acenar para a compreensão de que essas oscilações estão em conformidade com o tipo de trabalho realizado pelos sujeitos, já que os demais fatores permaneceram na média.

Em relação ao F3, habilidade de conversação e desenvoltura social, e ao F4, habilidade de exposição à desconhecidos e situações novas, percebem-se resultados nos parâmetros de referência. Pode-se inferir que a amostra da presente pesquisa não apresenta dificuldades importantes em situações sociais neutras de aproximação, supondo um bom conhecimento das normas sociais e de relacionamento no cotidiano (F3), bem como a presença de habilidade na abordagem de pessoas desconhecidas (F4).

Esses aspectos corroboram os achados do ZSC nos índices de repostas humanas $H$ puro e $S u m H$, que apresentaram-se nas expectativas normativas. Dessa forma, para que se tenha uma visão ainda mais completa da importância do Zulliger na análise do relacionamento interpessoal e produtividade, faz-se necessário aprofundar a discussão dos achados na correlação das variáveis de relacionamento do ZSC com os fatores do IHS-Del-Prette (Tabela 3).

Nessa linha de raciocínio, adverte-se que houve correlações estatisticamente significativas, de magnitude moderada e baixa, entre os instrumentos. A variável GHR apresentou uma correlação significativa de forma positiva e moderada com o escore geral de habilidades sociais GIHS. Essa correspondência é relevante, demonstra que na medida em que aumentam as respostas boas e interativas no ZSC também aumenta o repertório geral de habilidade social. Del Prette e Del Prette (2005) consideram que, quando as pessoas possuem um bom índice geral de habilidade social, elas têm uma melhor autoestima, conseguem interagir e ter melhores resultados no trabalho. Outro aspecto interessante é o fato dessa variável ter exibido correlações positivas significativas, embora de baixa magnitude, com mais três fatores do IHS-Del-Prette (F1, F3, F4). Isso revela uma tendência de aumentar essas habilidades sociais, quando aumenta o índice de respostas GHR.

Contrariamente, as variáveis PHR e $A G$ correlacionaram-se significativamente de forma negativa e moderada $(\mathrm{r}=-0,46$ e $\mathrm{r}=-0,44)$ com o $\mathrm{F} 5$, indicando que, diferente de GPHR, as variáveis PHR $e$ $A G$ aumentam, à medida que a habilidade de autocontrole da agressividade diminui ou vice-versa. Esses achados são compatíveis aos resultados de Di Domenico-Grazziotin \& Scortegagna (2012) que demonstraram que à medida que uma pessoa fornece mais respostas de má qualidade no ZSC, apresenta um menor percentil no F5 do IHS-Del-Prette, e confirmam que as variáveis GHR e PHR caminham em direções opostas (Viglione \& cols., 2003). 
Outro aspecto a ser destacado é a correlação significativa, positiva e baixa, entre a variável PHR e o fator F1. Para Del Prete e Del Prete (2005), esse fator está relacionado com o enfrentamento em situações de risco, sugerindo uma demanda maior de agressividade em comparação com a dos outros fatores. Portanto, a correlação do PHR, variável em que os índices de agressividade são consideráveis (Villemor Amaral \& Primi, 2009), com o F1 parece fazer sentido.

A variável GPHR apresentou correlação significativa de forma positiva e baixa com três fatores do IHS-Del-Prette (GIHS, F3, F5). Isso demonstra que essa variável, relacionada à capacidade mais madura do sujeito (Villemor-Amaral \& Primi, 2009), encontra concordância com habilidades sociais em um âmbito geral, com a conversação e desenvoltura social e o autocontrole da agressividade, respectivamente. Aspectos semelhantes foram demonstrados quando essa variável apresentou correlação significativa de forma positiva e moderada com o fator F3 e o GIHS (Di Domenico-Grazziotin \& Scortegagna, 2012).

É respeitável destacar que a variável SumT evidenciou correlação significativa positiva, embora de baixa magnitude, com o F2, sugerindo uma convergência do sombreado textura com o repertório da habilidade de autoafirmação e expressão de sentimento positivo. Vale lembrar, também, que o grupo estudado apresentou um repertório elaborado, acima da média nesse fator, e que no ZSC também houve uma maior incidência da variável $\operatorname{Sum} T$, que expressa a necessidade de contato (Villemor-Amaral \& Primi, 2009). Esses achados assinalam uma característica específica do grupo de dar e buscar receber mais afeto e, na função que ocupam, pode ser positivo. Este resultado corrobora a correlação significativa de forma positiva e baixa com o F2 encontrada em estudo preliminar (Di DomenicoGrazziotin \& Scortegagna, 2012).

Outras variáveis do relacionamento, como os conteúdos humanos, que demonstram o interesse pelos outros (Villemor-Amaral \& Primi, 2009), alcançaram correlações significativas com o IHS-Del-Prette. A variável Sum $H$ obteve uma correlação significativa, positiva e moderada com o fator F1, semelhante aos achados anteriores (Di Domenico Grazziotin \& Scortegagna, 2012). Isso denota que, à medida que aumenta a variável que demonstra o interesse pelas pessoas, aumenta a habilidade de enfrentamento com risco. O Sum $H$ também alcançou uma correlação significativa de forma positiva e baixa com os fatores GIHS e F4. Verifica-se, então, que à medida que o número de respostas humanas aumenta, o repertório geral de habilidades sociais e, especificadamente, o de autoexposição a desconhecidos e situações novas no IHS-Del-Prette, tendem a se elevar.

As respostas $H$ puro sugerem a presença de um interesse interpessoal mais visível, especificando uma identificação aberta e firme com pessoas (Villemor-
Amaral \& Primi, 2009). Essa variável obteve correlação significativa positiva e baixa, com o fator F3, e mostra a tendência em que à medida que as pessoas fornecem mais respostas humanas puras, aumenta o repertório da habilidade de conversação e desenvoltura social. Essa correlação já foi igualmente evidenciada (Di Domenico-Grazziotin \& Scortegagna, 2012) e assegura a validade do ZSC.

Quanto ao movimento ativo $a$, houve correlação significativa, positiva e moderada com o fator F1. Isso sugere que pessoas que denotam um incremento nas respostas de movimento ativo tendem a apresentar um aumento no repertório de habilidades, em situações de enfrentamento com risco. Achados semelhantes foram referidos por Ferreira e Villemor-Amaral (2005), ao verificarem correlações significativas de forma positiva e baixa entre movimento ativo e absorção, organização, registro e divulgação de conhecimento e apoiam os resultados de Di Domenico-Grazziotin e Scortegagna (2012) ao notarem atitudes mais ativas em amostra semelhante.

Considerando que o ZSC oferece tanto compreensões nomotéticas quanto idiográficas (Primi, Muniz \& Villemor Amaral, 2009), e que algumas das variáveis, neste estudo, não alcançaram correlações significativas, mas apresentaram coeficientes superiores a 0.20 , a interpretação qualitativa se faz necessária. Com esse propósito, a variável Sum $T$ teve correlação negativa, de baixa magnitude, não estatisticamente significativa com o F3 e o F5. Essas correlações, embora não tenham atingido o nível de significância esperado, auxiliam a pensar qualitativamente. Primeiro, por terem vindo em um sentido contrário, negativo, e segundo, por corroborarem teorias que afirmam que, quando muito aumentada, pode trazer prejuízos nas relações interpessoais (Exner \& Sendín, 1999; Ferreira \& Villemor-Amaral, 2005; Zudnic, 2003) e, neste caso, em habilidades como a conversação, a desenvoltura social, o autocontrole da agressividade em situações aversivas. Esses resultados apoiam a correlação negativa e moderada estatisticamente significativas com F5, encontradas em estudo anterior (Di DomenicoGrazziotin \& Scortegagna, 2012).

Em relação ao número de respostas $R$, não houve correlações estatisticamente significativas com os fatores do IHS-Del-Prette, apenas apresentaram uma correlação positiva de magnitude baixa, não estatisticamente significativa com F1. Apesar de estudos referirem que essa variável está relacionada a questões produtivas, cognitivas, motivacionais e colaborativas (Exner, 2003; Primi, Guntert \& Alchieri 2002; Villemor-Amaral \& Primi, 2009; Zudnic, 2003), não observou-se uma relação direta com habilidade social, em que pessoas menos conflitadas e com boa habilidade social são mais produtivas (Del Prete \& Del Prete, 2005). Todavia, se não é possível afirmar que o número de respostas esteve, em termos estatísticos, significativamente associado a habilidade social, 
também é correto dizer que não foi em direção contrária a essa habilidade.

Apesar disso, é interessante observar que o grupo estudado apresentou um número de respostas $R$ e as variáveis relacionamento na média $(\mathrm{M})$ nos parâmetros normativos, com destaque para questões afetivas e cooperativas no ZSC (Sum T e COP). O repertório de habilidade social, também situado na média, destacouse pelo afeto positivo e o autocontrole da agressividade (F2 e F5). Essa conexão entre os construtos reflete um nível adequado de relacionamento, habilidade social e, aliada a um bom número de respostas (motivação, energia, colaboração), parece propiciar uma produtividade satisfatória.

Portanto, pode-se perceber semelhanças entre os construtos relacionamento interpessoal e a habilidade social, o que sugere que, quando se correlacionem os dois, utilizando-se instrumentos distintos, mas confiáveis, se obtenha associações estatisticamente significativas. $O$ mesmo não acontece com produtividade e habilidade social, construtos diferentes e, então, mais difíceis de obter correlações estatisticamente significativas, considerando-se que o IHS-Del-Prette apresenta um número de respostas fechado, o que difere do ZSC.

\section{Conclusão}

Neste estudo, ficou demonstrado que GHR, GPHR, PURE H e SumH, que informam sobre o bom relacionamento e percepção interpessoal adequada, correlacionaram-se significativamente e de forma positiva com os fatores GIHS, F3, F5 e F1, respectivamente. Contrariamente, as variáveis $A G \mathrm{e}$ PHR, que podem indicar dificuldades de relacionamento, apresentaram correlações significativas de forma negativa com o fator F5.

O número de respostas do ZSC não obteve correlação estatisticamente significativa com os fatores do IHS-Del-Prette, mas qualitativamente denotou uma conexão. Portanto, este estudo trouxe resultados valiosos ao ratificar a validade do Zulliger e, ainda, ao revelar as características da amostra estudada. Por fim, justifica-se a necessidade de seguimento de estudos de validade do Zulliger pela importância desse teste, que permite a avaliação da personalidade confiável, mais rápida, e pela insuficiência de estudos brasileiros com amostras diversificadas e ampliadas.

\section{Referências}

Angélico, A. P., Crippa, J. A. S. \& Loureiro, S. R. (2012). Utilização do Inventário de Habilidades sociais no diagnóstico do transtorno de ansiedade social. Psicologia: Reflexão e Crítica, 25(3), 467-476.

Bandeira, M., Costa, M. N., Del Prette, Z. A. P., Del Prette, A. \& Gerk-Carneiro, E. (2000). Qualidades psicométricas do Inventário de Habilidades Sociais
(IHS): estudo sobre a estabilidade temporal e a validade concomitante. Estudos de Psicologia, 5(2), 401-419.

Burlamaque, A. V., Zardo, C. E., Secco, A. C. \& Scortegagna, S. A. (2012). Panorama brasileiro de publicações sobre o teste Zulliger no período de 2002-2012. Em L. J. G. Barcellos, L. R. dos Santos, M. Eichelberger \& C. Chais (Orgs.). Anais da XXII Mostra de Iniciação Científica. Passo Fundo: Editora Universidade de Passo Fundo.

Conselho Federal de Psicologia. (2003). Manual de elaboração de documentos decorrentes de avaliação psicológica. [Instituído pela Resolução CFP $\mathrm{n}^{\circ}$ 007/2003]. Disponível em http:// www. pol. org.b r/pol /export /sites/ default/ pol / legislacao/legislacaoDocumentos/resolucao2003_ 7.pdf.

Conselho Federal de Psicologia. (2003b). Resolução CFP $n^{\circ}$ 002/2003. Disponível em http:// www. pol. org. br/ pol/ export /sites /default/ pol/ legislacao/ legislacao Documentos/resolucao2003_02.pdf.

Conselho Federal de Psicologia. (2005). Resolução CFP $n^{\circ} \quad 010 / 2005$. Disponível em http://www.pol.org.br/pol/export/sites/ default /pol/ legislacao/ legislacao Documentos /resolucao 2005_10.pdf.

Del Prette, Z. A. P. \& Del Prette, A. (2005). Inventário de Habilidades Sociais (IHS-Del- Prete): manual de aplicaşão, apuração e interpretação ( $3^{a}$ ed.). São Paulo: Casa do Psicólogo.

Del Prette, Z. A. P. \& Del Prette, A. (2010). Habilidades sociais e análise do comportamento: proximidade histórica e atualidades. Revista Perspectivas, 1(2), 104-115. Disponível em http://www.revistaperspectivas.com.br.

Di Domenico-Grazziotin, J. B. \& Scortegagna, S. A. (2012). Zulliger e habilidade social: evidências de validade no contexto empresarial. Psicologia: Reflexão e Crítica, 25(1), 69-78.

Dilchert, S., Ones, D. S., Visweswaran, C. \& Deller, J. (2006). Response distortion in personality measurement: born to deceive, yet capable of providing valid assessments? Psychology Science, 48(3), 209-225. Disponível em http://www.pabstpublishers. de/ psychology-science/32006/ps_3_2006_209-225.pdf.

Exner, J. E. Jr. (2003). The Rorschach: a comprehensive system (Vol. I: Basic foundations). Nova Iorque: Wiley \& Sons.

Exner, J. E. Jr. \& Sendin, C. (1999). Manual de interpretação do Rorschach para o sistema compreensivo. São Paulo: Casa do Psicólogo. 
Fazendeiro, H. \& Novo, R. F. (2012). Versão coletiva do teste de Zulliger segundo o sistema compreensivo de Rorschach. Avaliação Psicológica, 11(3), 407-422.

Ferreira, M. E. A. \& Villemor-Amaral, A. E. (2005). O teste de Zulliger e avaliação de desempenho. Paidéia, 15(32), 367-376. Disponível em http:// www. scielo. br/ pdf/ paideia /v15n32/06.pdf.

Franco, R. R. C, Cardoso, L. M., Villemor-Amaral, A. E. \& Primi, R. (2009). Estudos de normatização, precisão e validade. Em A. E. Villemor-Amaral \& R. Primi (Orgs.). Teste de Zulliger no sistema compreensivo ZSC: forma individual. São Paulo: Casa do Psicólogo.

Franco, R. R. C. \& Villemor-Amaral, A. E. (2009). Ensaios de convergência entre provas de personalidade: Zulliger-SC e Pfister (Tese de Doutorado). Itatiba, SP: Universidade de São Francisco.

Franco, R. R. C. \& Villemor-Amaral, A. E. (2012). Validade incremental do Zulliger e do Pfister no contexto de toxicomania. Psico-USF, 17(1), 73-83.

Guilland, R. \& Monteiro, J. K. (2010). Jovem em situação de desemprego: Habilidades Sociais e bem-estar psicológico. Psicologia: Teoria e Prática, 12(3), 149-163.

Meade, A. W. (2004). Psychometric problems and issues involved with creating and using ipsative measures for selection. Journal of Occupational and Organizational Psychology, 77, 531-552.

Muniz, M., Machado, M. A., Villemor-Amaral, A. E. \& Primi, R. (2009). Precisão do Zulliger no Sistema Compreensivo. Em A. E. Villemor-Amaral \& R. Primi. (Orgs.). Teste de Zulliger no sistema compreensivo ZSC: forma individual. São Paulo: Casa do Psicólogo.

Nunes, C. (2011). Importância da especificação dos contextos de aplicação e propósitos nos manuais de testes psicológicos. Em Conselho Federal de Psicologia (Org.). ano da avaliaşão psicológica - textos geradores (pp. 59-63). Brasilia, DF: CFP.

Nunes, C. H. S. S. \& Primi, R. (2010). Aspectos técnicos e conceituais da ficha de avaliação dos testes psicológicos. Em Conselho Federal de Psicologia (Org.). Avaliação psicológica: diretrizes na regulamentação da profissão (pp. 101-127). Brasilia, DF: CFP.

Primi, R., Guntert, A. E. V. A. \& Alchieri, J. C. (2002). Um estudo correlacional entre a bateria de provas de raciocínio (BPR5, R1 e o TNRV) com o teste de Zulliger. Psico (PUCRS), 33(1), 53-76
Primi, R., Muniz, M. \& Villemor-Amaral, A. E. (2009). Validade do Zulliger no sistema compreensivo. Em A. E. Villemor-Amaral \& R. Primi. (Orgs.). Teste de Zulliger no sistema compreensivo ZSC: forma individual. São Paulo: Casa do Psicólogo.

Santana, P. R., Cardoso, L. \& Villemor-Amaral, A. E. (2011). Instituindo a nota $Z$ por meio do modelo de Rasch para o teste Zulliger. Resumos do $V$ Congresso Brasileiro de Avaliação psicológica: Avanços e Desafios. Bento Gonçalves, RS.

Silva, C. A. (2004). Habilidades sociais em fusão de organizacoêes: uma estratégia preventiva do estresse (Dissertação de Mestrado). São Bernardo do Campo, SP: Universidade Metodista de São Paulo - UMESP.

Viglione, D. J., Perry, W., Jarsak, D., Meyer, G. \& Exner, J. E. (2003). Modifyng the Rorschach human experience variable to create the human representational variable. Journal of Personality Assessment, 8(1), 64-73.

Villemor-Amaral A. E. \& Cardoso, L. M. (2011). Evidências de validade convergente do tipo de vivência (EB) no teste de Zulliger SC. Resumos do $V$ Congresso Brasileiro de Avaliação Psicológica: Avanços e Desafios. Bento Gonçalves, RS.

Villemor-Amaral, A. E. \& Primi, R. (2009). Teste de Zulliger no sistema compreensivo ZSC: Forma individual. São Paulo: Casa do Psicólogo.

Villemor-Amaral, A. E., Machado, M. A. S. \& Noronha, A. P (2009). O Zulliger no sistema compreensivo: Um estudo de fidedignidade. Psicologia Ciência e Profissão, 29(4), 656-671.

Zudnic, L. A. (2003). El teste Zulliger en la evaluación psicológica de personal: Aportes del sistema compreensivo de Exner. ( $2^{\mathrm{a}}$ ed., Cuadernos de Evaluación Psicológica). Buenos Aires: Paidós.
Recebido em 08/05/2012

Revisado em 08/02/2013

Aprovado em 22/05/2013 
Sobre as autoras:

Jucelaine Bier Di Domenico Grazziotin é aluna de Mestrado em Envelhecimento Humano da Universidade de Passo Fundo.

Silvana Alba Scortegagna é doutora em Avaliação Psicológica pela Universidade São Francisco e docente da Graduação em Psicologia e da Pós-Gradução Stricto Sensu em Envelhecimento Humano da Universidade de Passo Fundo.

\section{Contato com as autoras}

Programa de Pós-Graduação Stricto Sensu em Envelhecimento Humano Universidade de Passo Fundo Campus I Bairro São José BR 285 km 171

CEP 99052-900 - Cx. Postal 611 - Passo Fundo/RS

Email: jucelainegraz@terra.com.br silvanalba@upf.br 